\title{
Educación 4.0 como respuesta a la Industria 4.0: un estudio analítico-descriptivo
}

\author{
Carlos Silverio Huerta Jiménez \\ carlos@usmexfusion.org \\ Doctorante del Doctorado en Investigación \\ e Innovación Educativa de la \\ Benemérita Universidad Autónoma de Puebla \\ Marco Velázquez Albo \\ marcovelazquez_buap@yahoo.com.mx \\ Profesor Investigador del Doctorado en \\ Investigación e Innovación Educativa de la \\ Benemérita Universidad Autónoma de Puebla \\ y Decano del Colegio de Historia
}

\section{RESUMEN}

El objetivo de este estudio de corte teórico analítico-descriptivo es hacer un análisis que conteste a la pregunta ¿de qué forma la industria 4.0 viene a cambiar las formas en que el aprendizaje toma lugar? Se reflexiona sobre la propuesta de "flujos de aprendizaje" como avenidas continuas y omnipresentes de adquisición de conocimiento y desarrollo de habilidades; y se realiza un análisis de los fundamentos de la Educación 4.0. Se concluye que esta educación supone un cambio de paradigma en relación con el rol del docente, el rol de los alumnos, el rol del aula, y el rol de los espacios no áulicos. En una educación 4.0, el aprendizaje toma lugar en todo momento. Los alumnos navegan en flujos de aprendizaje que van más allá los ambientes escolares.

Palabras clave: Industria 4.0; Educación 4.0; Enseñanza-Aprendizaje 


\title{
Education 4.0 as a response to Industry 4.0: an analytical-descriptive study
}

\begin{abstract}
The purpose of this analytical-descriptive theoretical study is to make an analysis that answers the question, how does Industry 4.0 change the ways in which learning takes place? It reflects on the proposal of "learning flows" as continuous and omnipresent avenues for the acquisition of knowledge and development of skills; and an analysis of the fundamentals of Education 4.0 is carried out. It is concluded that this education represents a paradigm shift in relation to the role of the teacher, the role of the students, the role of the classroom, and the role of non-classroom spaces. In a 4.0 Education, learning takes place at all times. Students navigate learning flows that go beyond school settings.
\end{abstract}

Keywords: Industry 4.0; Education 4.0; Teaching-Learning

Artículo recibido: 25 enero 2021 Aceptado para publicación: 28 febrero2021 Correspondencia: carlos@ usmexfusion.org Conflictos de Interés: Ninguna que declarar 


\section{INTRODUCCIÓN}

¿De qué forma la Industria 4.0 viene a cambiar las formas en que el aprendizaje toma lugar? Esta pregunta motivó el presente estudio. Se inicia con una explicación de la Industria 4.0, la cual presenta un nuevo paradigma de trabajo y un nuevo paradigma de educación. La respuesta a este nuevo paradigma es la Educación 4.0. El aprendizaje toma lugar en todo momento, y no se requiere necesariamente de un lugar específico: el aula. Se explica cuáles son los fundamentos de una educación 4.0 y se reflexiona sobre la labor del docente: un facilitador más que un transmisor de conocimientos. La educación toma lugar por medio de flujos de aprendizaje y no solo en instituciones educativas o en ambientes áulicos presenciales o a distancia. Ante esta realidad compleja ya no es posible ver a la educación desde perspectivas reduccionistas o simplistas. Se explica que es imperativo ver a la Educación 4.0 insertada en una realidad en la que la fusión de tecnologías hace confusa la separación entre lo biológico, lo físico, y lo digital. En décadas pasadas se pensaría como imposible que las disciplinas de estas áreas pudieran interactuar. En un futuro no muy lejano se podrán construir órganos de seres humanos con impresoras especializadas 3-D (WEF, 2015). Para lograr una educación acorde a esta realidad, es necesario analizar con una mirada analítica los fundamentos de la Educación 4.0. Los elementos de esta introducción se desarrollan en este texto para dar respuesta a la pregunta de investigación.

\section{MÉTODO Y MATERIALES}

El presente estudio es de corte teórico analítico-descriptivo. La metodología para el estudio parte de un análisis documental de fuentes primarias más citadas en la literatura académica que se relacionan con la Industria 4.0 (Shwab, 2016; World Economic Forum, 2015) y con la Educación 4.0 como el Instituto para el Futuro (2013) y el autor Peter Fisk (2017) creador de The Genius Works. Bajo un enfoque constructivista, se reflexiona sobre la propuesta de "flujos de aprendizaje" como avenidas continuas y omnipresentes de adquisición de conocimiento y desarrollo de habilidades; y se realiza un análisis de los fundamentos de la Educación 4.0.

\section{RESULTADOS Y DISCUSIÓN}

\section{¿CÓMO LLEGAMOS A LA EDUCACIÓN 4.0?}

La educación 4.0 surge a partir de la llamada cuarta revolución industrial o Industria 4.0. Shwab (2016) explica de la siguiente manera los cambios que han marcado las 
transiciones entre las diferentes revoluciones industriales: la industria 1.0 se basaba en la mecanización de procesos mediante equipo cuya fuerza era generada con agua o vapor. La industria 2.0 usó la energía eléctrica para la masificación de la producción. La industria 3.0 se basaba en el uso de aparatos electrónicos y de la tecnología de la información para automatizar la producción. La industria 4.0 se caracteriza por la fusión de tecnologías que hacen que lo biológico, lo físico y lo digital se unan y sea difícil marcar una línea donde uno termina y el otro comienza.

Estas fusiones de lo biológico-físico-digital las podemos encontrar en los avances en áreas como la biotecnología, la inteligencia artificial, el internet de las cosas, la robótica, la impresión en 3-D y los vehículos autónomos, entre otras. El Foro Económico Mundial (WEF, 2015) presenta ejemplos de estos avances o "puntos de inflexión" que ocurrirán antes del año 2025: el 90\% de la población mundial usarán internet y el $80 \%$ tendrá presencia digital; estará disponible comercialmente el primer teléfono móvil implantable; el 90\% de la población tendrá almacenamiento sin límite de capacidad y sin costo en la nube; más del 50\% del tráfico de internet en los hogares será para electrodomésticos y dispositivos; el 10\% de las gafas de lectura estarán conectadas a internet; el 10\% de las personas usarán ropa conectada a internet; se hará el primer trasplante de un hígado creado con una impresora 3-D; habrá el primer automóvil en producción creado con impresoras 3-D.

La Industrial 4.0 tiene repercusiones en todos los ámbitos, y la educación no es la excepción. Es por esto que ahora se necesita repensar una educación que responda a esta realidad. Una educación 4.0 usa a las tecnologías digitales como principales medios de aprendizaje y comunicación, usa el internet como un espacio global común de educación, y aprovecha el conocimiento generado a nivel mundial; los estudiantes se vinculan a nivel internacional para educarse y trabajar, usan procesos de enseñanza-aprendizaje que van más allá de lo que aprende en las aulas, usan un mayor número de idiomas que sólo su lengua materna, requieren de fuertes conocimientos computacionales, aprovechan la tecnología para facilitar los procesos de aprendizaje y manejo del conocimiento, y requieren de maneras de pensar que entiendan la transdisciplinariedad, entre otros. Parece que el mundo se vuelve plano y que sus complejidades son parte de la vida educativa y laboral de las personas. Estos procesos toman lugar en flujos de aprendizaje y de trabajo. Las oficinas, fábricas, y las universidades son parte de estos flujos, pero no son 
necesariamente los lugares únicos o más importantes. El trabajo y la educación toman lugar en cualquier lugar, tiempo, y en variados contextos.

\section{DE INSTITUCIONES EDUCATIVAS A FLUJOS DE APRENDIZAJE}

Por décadas (o siglos), se ha asumido que es necesario ir a la escuela para aprender. Los profesores, el currículo, los materiales, las aulas, y las metodologías de enseñanza forman parte de los actores y actividades dentro de las escuelas. Sin embargo, en el siglo XXI lo opuesto está sucediendo: los alumnos aprenden cada vez más de manera autónoma y lo hacen cada vez más fuera de las aulas o las escuelas. ¿Será que lo que sucede en las aulas no es coherente con lo que sucede afuera en la vida real?, ¿será que las aulas podrían representar para los alumnos obstáculos para aprender en lugar de lugares de apalancamiento para su aprendizaje? La realidad de los estudiantes y trabajadores en la vida actual se desarrolla a una velocidad más acelerada que lo que sucede en las aulas; y la tecnología que la gente y los alumnos usan supera a la tecnología de las aulas.

El Instituto para el Futuro (IFTF, 2013) explica que la educación 4.0 va más allá de los ambientes áulicos y de las instituciones educativas. Para este organismo no gubernamental, la educación actual se da por medio de "flujos de aprendizaje" y no por medio de instituciones educativas. La educación ya no toma lugar en un sitio y en un tiempo específico. La educación se lleva a cabo en un flujo que tiene como características la ubicuidad, la flexibilidad, la transdisciplinariedad, y la adaptabilidad.

Este instituto justifica esta realidad en relación con el aprendizaje y el trabajo a través de seis escenarios que ya son comunes en el presente y lo serán más en el futuro inmediato: Primero, el aprendizaje está al alcance de la mano. El acceso a la información y el aprendizaje ya no se dan exclusivamente en bibliotecas, salones de clase, o laboratorios. En el continuo vivir, en las experiencias diarias, se tiene acceso y se interactúa con el conocimiento: al caminar, al ir en el autobús, al estar sentado en casa o en un parque. Si se necesitara de un tutor o un maestro, se puede tener de inmediato por medio de un dispositivo de comunicación como un teléfono inteligente, una tableta, o una laptop.

Segundo, el aprendizaje ya se puede llevar a cabo mediante materiales curados (revisados y elegidos por su calidad) disponibles a través de la nube. El flujo del conocimiento toma lugar en plataformas educativas que ofrecen cursos como los Massive Open Online Courses (MOOC). Estos cursos son confiables, ya que son creados por profesores de reconocidas instituciones de educación. Toda la interacción se da a través de plataformas 
educativas. Los buscadores de información se vuelven cada vez más sofisticados con la capacidad de encontrar y analizar contenido relevante. En el futuro cercano la mayoría de la gente trabajará en la nube teniendo acceso a información personalizada desde cualquier dispositivo. Las diferentes aplicaciones para manejo de la información funcionarán cada vez más en coordinación con la nube de manera automática.

Tercero, los materiales para aprender se ofrecen en diferentes formatos. La información y los contenidos se presentan en formatos que van más allá del texto o el hipertexto. Simuladores, videos, audios, fotografías, etc. se convierten en fuentes de información disponible en el internet. El contenido tiende a ofrecerse como abierto o gratis. Los materiales impresos son menos usados debido a que las nuevas generaciones interactúan con mayor facilidad con textos digitales que contienen hipertexto, imágenes y gráficos interactivos.

Cuarto, los servicios educativos se ofrecen en línea. Se crean las primeras universidades $100 \%$ en línea, se crean nuevas formas de obtener un grado académico, nuevas formas de reconocer credenciales, nuevas formas de certificar habilidades o conocimientos, nuevas formas de apoyo al aprendizaje como tutores en línea. Estos servicios educativos se esfuerzan por demostrar su calidad y la validez de su oferta educativa. La reputación de estas instituciones se gana o se pierde a través de: usuarios que "suben" comentarios y opiniones en relación con su experiencia usando estas modalidades de aprendizaje, la calidad de los servicios virtuales, el reconocimiento o acreditación que tienen, y el valor que les otorgan los empleadores y la comunidad en general.

Quinto, existen más modalidades para el trabajo y el estudio. La colaboración virtual rompe las barreras de la distancia y el trabajo se puede llevar a cabo en grupos colaborativos. Estos grupos persiguen determinados objetivos para poder estudiar o trabajar en modalidades de colaboración por medio de sistemas sincrónicos como videoconferencias o chats en tiempo real, o asincrónicos como foros o grupos que trabajan en plataformas para implementación de proyectos. Ya no existen los horarios de clase o de trabajo en bloque (de 9 am a 5 pm, por ejemplo). El trabajo se logra con base en objetivos o proyectos que tienen fechas específicas de término y se llevan a cabo por medios virtuales. 
Sexto, las tecnologías potencian las capacidades humanas para trabajar y aprender. Las máquinas y el software inteligente se vuelven cada vez más omnipresentes en nuestras vidas diarias. Los doctores hacen cirugías a distancia, los profesores dan clases a distancia. Las máquinas ayudan a potenciar la memoria, la vista, la capacidad analítica de una persona, las máquinas dan retroalimentación del trabajo o del aprendizaje de manera inmediata.

Estos seis escenarios dan una clara idea de que la educación ya no requiere de un aula y un espacio específico en el tiempo. El aprendizaje es ubicuo: está en todas partes y al mismo tiempo para todos por medios digitales y el internet. La cobertura de internet avanza rápidamente en el mundo. Como ejemplo, Google trabaja para tener globos con módems inalámbricos que provean de conexiones $\mathrm{Wi}$-Fi a todas las regiones del mundo, especialmente a las alejadas de las ciudades. Para este proyecto, Google usó habilidades de su equipo como la flexibilidad, la transdisciplinariedad, y la adaptabilidad. Tenían que tomar en cuenta las condiciones meteorológicas de todas las regiones del mundo para que los globos se mantuvieran constantemente en el aire, a una altura específica, y que cubrieran una región específica (LOON, s.f).

Cada vez más, el aprendizaje toma lugar considerando intereses específicos de las personas y se suministra al ritmo que las mismas lo requieran. Se da la transdisciplinariedad con más frecuencia ya que las personas percatan de la necesidad de saber de muchas cosas como una ventaja en relación con las personas que se especializan sólo en un tema o área. Saber programar por ejemplo será una actividad común entre todos los campos, igualmente lo será saber pensar y entender la complejidad (Morin et. al., 2002) la literacidad digital, y el manejo de todo tipo de gadgets que apoyen el aprendizaje, entre otros. Estas habilidades parecerían ser sólo pertinentes para ingenieros computacionales, programadores o diseñadores digitales, pero ya son habilidades que podríamos considerar como transversales a todas las profesiones. Estas habilidades son fundamentales, especialmente en la educación 4.0.

\section{FUNDAMENTOS DE LA EDUCACIÓN 4.0: UN ANÁLSIS}

¿Es posible que los profesores actuales, además de dedicarse a compartir conocimientos y desarrollar habilidades propias de sus materias, tienen también que apoyar el desarrollo de las habilidades para una educación 4.0? A partir de las realidades expuestas en la sección anterior suponemos que así debe ser. Una educación 4.0 presenta un cambio de 
paradigma. Se requiere cambiar la visión que se tiene sobre el aprendizaje. Para Fisk (2017), esta nueva visión promueve que los estudiantes aprendan no sólo las habilidades y los conocimientos, sino también que identifiquen las fuentes para desarrollar estas habilidades y adquirir conocimiento. El aprendizaje se centra aún más en el estudiante y el seguimiento a su desempeño se lleva a cabo a través de la personalización basada en datos sobre su desempeño previo. Los compañeros se convierten en algo muy importante para el aprendizaje. Aprenden juntos y entre ellos, mientras que los profesores asumen un rol de facilitadores en el proceso de aprendizaje.

Fisk propone ocho características principales para la Educación 4.0. La primera es que el aprendizaje puede llevarse a cabo en cualquier lugar y a cualquier hora. Las herramientas de aprendizaje electrónico (e-learning) ofrecen grandes oportunidades para el aprendizaje remoto y al ritmo del estudiante. El método del aula invertida (flipped classroom) también es importante ya que permite que el aprendizaje interactivo tome lugar durante la clase, mientras que las partes teóricas se pueden revisar fuera del tiempo de clase. Se requiere de los estudiantes una capacidad para aprender a aprender, ya que cada vez más trabajan por su cuenta, ya sea solos o con sus compañeros para aprender fuera del aula. El rol del profesor durante el curso es de acompañamiento a los alumnos para apoyarlos a desarrollar las habilidades y adquirir los conocimientos esperados. El docente no solo cumple con esta labor en el aula, sino también a través de plataformas educativas digitales, cursos y materiales en línea, el involucramiento de sus alumnos con otros alumnos de otras partes del país o del mundo; potenciando así las habilidades y conocimientos de sus alumnos.

La segunda característica es que el aprendizaje estará personalizado para cada estudiante. Recibirán tareas más complejas a partir de que hayan mostrado dominio en tareas más básicas. El avance en el aprendizaje no tiene que darse necesariamente en el mismo orden y al mismo tiempo para todos. El refuerzo y reciclamiento de los conocimientos promueven que los alumnos tengan más confianza en sí mismos y en sus habilidades académicas. Un ejemplo de esta modalidad de aprendizaje la ofrece Khan Academy (https://www.khanacademy.org), una plataforma de aprendizaje de acceso abierto cuyo currículum siguen muchas escuelas en el mundo. La plataforma ayuda a los docentes a diseñar "learning paths" o rutas de aprendizaje que se adapten al ritmo y al dominio de los conocimientos por parte de los alumnos. De esta manera ya no se imparten clases en 
donde se les enseña a todos lo mismo independientemente de si están listos o no para aprender "lo que sigue". De la misma manera se evalúa. Ya no existe un examen que intente evaluar bajo los mismos parámetros a todos los alumnos en un momento determinado.

La tercera característica es que los estudiantes tienen la opción de determinar cómo quieren aprender. Aunque los estudiantes tienen que lograr los objetivos de aprendizaje establecidos en el currículo, tienen la libertad para elegir herramientas o técnicas de aprendizaje. Para facilitar este tipo de aprendizaje, los profesores pueden elegir el aprendizaje mixto (presencial y en línea), el aula invertida, y el BYOD ("Bring your own device" o "Trae tu propio dispositivo "). Esta última modalidad es interesante ya que los alumnos cada vez más trabajan en la nube y se sienten más cómodos con la manera en cómo su equipo se comporta con las diferentes aplicaciones, plataformas de aprendizaje o software que ellos han seleccionado como apoyo para sus procesos de aprendizaje.

La cuarta característica es que los estudiantes serán expuestos a más aprendizaje a través de proyectos. Se requiere que los estudiantes apliquen sus conocimientos y habilidades para completar uno o dos proyectos a la vez. Al involucrarlos en un proyecto, los alumnos practican sus habilidades organizacionales, colaborativas, y de manejo de tiempo. Estas habilidades son muy útiles para la vida profesional y personal. En este sentido, los alumnos pueden también tener como proyecto enseñarse a sí mismos en equipos. Esto no necesariamente significa retirar al profesor completamente de su figura, sino más bien significa que el profesor adoptaría otras funciones para lograr que los alumnos alcancen el objetivo de enseñarse a sí mismos y entre ellos mismos. En otras palabras, los estudiantes son apoyados con estrategias para que sean ellos mismos, los que estén a cargo de sus procesos de enseñanza-aprendizaje y que puedan enseñar a sus compañeros. Desde hace más de tres décadas, Cohen et al. (1982) han encontrado dos principales beneficios de que los estudiantes faciliten el aprendizaje a sus compañeros: 1) que aprender de otros estudiantes coadyuva para un mejor entendimiento de los contenidos y 2) que aprender de otros estudiantes promueve una actitud más abierta a aprender los contenidos de un curso.

La quinta característica es que los estudiantes serán expuestos cada vez más a aprendizaje a través de la práctica por medio de trabajo de campo como las estadías, servicio social, y proyectos colaborativos. Esto permite a los estudiantes practicar habilidades de 
relaciones humanas y de interacción cara a cara ya sea en persona o por medios virtuales. Esta no es una actividad nueva. El aprendizaje a través de la práctica forma parte de sistemas educativos de nivel medio superior y superior en muchos países del mundo desde hace décadas. Lo interesante aquí es ver cómo las Tecnologías de Información y Comunicación (TIC), el internet, la realidad virtual entre otras, potencian estas actividades.

La sexta característica es que los estudiantes serán expuestos a interpretación de datos en la que se requerirá aplicar conocimientos teóricos a los números y datos, así como usar sus habilidades de razonamiento para hacer inferencias basadas en la lógica y patrones a partir de sets de datos. La parte matemática manual de operaciones se volverá irrelevante ya que las computadoras harán los análisis estadísticos y predicciones de patrones futuros. Este punto es en donde los seres humanos cada vez más buscan las maneras de cómo las computadoras puedan hacer los trabajos de "razonamiento" que en su momento tenían que hacer las personas. Dejar a las computadoras organizar nuestras actividades, organizar la información que es de nuestro particular interés, ofrecernos reportes ejecutivos de comportamientos de ciertos procesos educativos o laborales, comprobar la confiabilidad de las fuentes, predecir posibles patrones de comportamiento económico, etc., le permite al ser humano navegar y procesar la gran cantidad de información que existe.

La séptima característica es que los estudiantes serán evaluados de manera diferente. Además de evaluar el conocimiento, se evalúa el desempeño al estar trabajando en los proyectos. Los exámenes de preguntas y respuestas se vuelven casi irrelevantes e insuficientes. Parecería que esto ya no necesita mencionarse, ya que se esperaría que estas prácticas ya fueran la norma en la actualidad. Pero la realidad es diferente. Todavía en muchas aulas la evaluación se da únicamente de forma cuantitativa y no cualitativa (o formativa). En muchas aulas los exámenes escritos aún representan el mayor valor en los criterios de evaluación.

La octava característica es que la opinión de los estudiantes se tomará en consideración para el diseño y actualización del currículo. Sus comentarios ayudan a los diseñadores curriculares a mantener el currículo actualizado y útil. Esta no es necesariamente una novedad pero en la práctica no se lleva a cabo. La voz de los estudiantes debería escucharse en la toma de decisiones de todos los procesos educativos. 
Desafortunadamente, en sistemas que supuestamente pretenden ayudar a los alumnos a aprender, la voz de los alumnos no existe. Esto equivale a ir al doctor y sin que el paciente haya hablado, el doctor le prescriba los medicamentos que "necesita". En la actualidad hay una línea de investigación llamada "Students as Partners" (Estudiantes como Compañeros) aplicada al aprendizaje global. Una de las principales investigadoras es Wendy Green (2019), quien en sus estudios ha encontrado que cuando los alumnos trabajan con el profesor para promover el aprendizaje global tanto dentro como fuera del aula los resultados son mejores. De esta manera demuestra que la práctica tradicional en la que solo el profesor decide cómo se va a llevar a cabo el aprendizaje no es la más satisfactoria y exitosa.

A partir de estos ocho escenarios se observa cómo en este cambio de paradigma se hace un empoderamiento del estudiante. El poder que el profesor mantuvo por décadas o siglos dentro del aula se les otorga ahora a los alumnos. Los alumnos deciden cómo aprender y desarrollar sus habilidades, colaboran para lograr sus objetivos, son parte de la creación de los planes de estudio, hacen uso de las tecnologías de manera que generaciones anteriores no se hubieran imaginado, forman parte de las evaluaciones, etc. El profesor se convierte en un facilitador y apoya a los estudiantes a desarrollar sus capacidades y conocimientos. La evaluación formativa toma más importancia que la evaluación sumativa. Los docentes se enfocan más en lo cualitativo del aprendizaje que en lo cuantitativo. En la medida en que los profesores se convenzan de que el modo de aprendizaje tiene que cambiar y la celeridad en que tomen cartas en el asunto será la medida en que los estudiantes potencien sus capacidades y conocimientos de manera innovadora. Como resultado de este cambio, los niveles de frustración que desarrollan los estudiantes y la aversión a la educación tradicional disminuirán.

\section{CONSIDERACIONES FINALES}

Una de las formas en las que se puede concebir la realidad actual es que la misma está afectada por la industria 4.0. La industria 3.0 que se basa en el uso de aparatos electrónicos y de la tecnología de la información para automatizar la producción está siendo rebasada por una industria 4.0 que se caracteriza por la fusión de tecnologías que hacen confusa la separación entre lo físico, lo digital, y lo biológico. En décadas pasadas se pensaría como imposible que las disciplinas de estas áreas pudieran interactuar. Esta complejidad hace evidente que mantener a las áreas del conocimiento separadas ya no es posible. La 
transdisciplinariedad, el análisis complejo, y el trabajo especializado en equipo, son habilidades que los profesores deben desarrollar en sus alumnos. Esta educación supone un cambio de paradigma en relación con el rol del docente, el rol de los alumnos, el rol del aula, y el rol de los espacio no áulicos. En una educación 4.0, el aprendizaje toma lugar en todo momento y no se requiere necesariamente de un lugar específico: el aula. Los alumnos se encuentran en flujos de aprendizaje durante su vida que van más allá los ambientes escolares. El profesor es un facilitador, un creador de estrategias de aprendizaje innovadoras, un organizador de actividades grupales a partir de proyectos que representen retos para los estudiantes, un líder que asume que su liderazgo debe estar orientado a que sus alumnos sean también líderes y que sean empoderados para auto-educarse y apoyar en el proceso de enseñanza-aprendizaje de sus compañeros.

\section{REFERENCIAS BIBLIOGRAFICAS}

Cohen, P., Kulik, J. y Lin, C. (1982). Educational Outcomes of Tutoring: A metaanalysis of findings, American Educational Research Journal, 19(2), 237-248, doi: $10.3102 / 00028312019002237$

Fisk, P. (2017). Education 4.0 ... the future of learning will be dramatically different, in school and throughout life. Recuperado de https://www.thegeniusworks.com/2017/01/future-education-young-everyonetaught-together/

Green, W. (2018). Engaging Students as Partners in Global Learning: Some Possibilities and Provocations, Journal of Studies in International Education, 23(1), 10-29. doi: 10.1177/1028315318814266

Morín, E., Ciurana, E, y Domingo, R. (2002). Educar en la era planetaria. El pensamiento complejo como Método de aprendizaje en el error y la incertidumbre. Valladolid: UNESCO.

IFTF (2013). From Educational Institutions to Learning Flows. California: Institute for the Future. Recuperado de http://www.iftf.org/uploads/media/SR-1580IFTF_Future_of_Learning_01.pdf

LOON (s.f). Navigating the winds. Recuperado de https://loon.com/technology

Schwab, K. (2016). The Fourth Industrial Revolution: what it means, how to respond. Global Agenda: Fourth Industrial Revolution. (World Economic Forum Blog). 
Recuperado de https://www.weforum.org/agenda/2016/01/the-fourth-industrialrevolution-what-it-means-and-how-to-respond/

WEF (2015). Deep Shift. Technology Tipping Points and Societal Impact. Ginebra: World Economic Forum. Recuperado de http://www3.weforum.org/docs/WEF_GAC15_Technological_Tipping_Points_rep ort_2015.pdf 